\title{
Improvement of Detection Limit of Nanomechanical Deflection Using Optical Interferometry for Label-free Molecular Detection
}

\author{
Satoshi Maruyama, ${ }^{1,2 \dagger}$ Yong Joon Choi,${ }^{1 \dagger}$ Kazuhiro Takahashi, ${ }^{1,3^{*}}$ and Kazuaki Sawada ${ }^{1}$ \\ ${ }^{1}$ Department of Electrical and Electronic Information Engineering, Toyohashi University of Technology, \\ 1-1 Hibarigaoka, Tempaku, Toyohashi, Aichi 441-8580, Japan \\ ${ }^{2}$ AIST-TUT Advanced Sensor Collaborative Research Laboratory, Toyohashi University of Technology, \\ 1-1 Hibarigaoka, Tempaku, Toyohashi, Aichi 441-8580, Japan \\ ${ }^{3}$ JST Precursory Research for Embryonic Science and Technology (PRESTO), \\ Tokyo 102-0076, Japan
}

(Received December 27, 2018; accepted July 17, 2019)

Keywords: Fabry-Perot interferometric sensor, MEMS, optical, biosensor, antigen-antibody reaction

Herein, we report an improved minimum detectable displacement on a surface stress biosensor using optical interferometry, allowing for the detection of a low biomarker concentration via antigen-antibody reaction. The sensor is composed of a submicronthick freestanding polymethyl methacrylate (PMMA)/parylene-C membrane on a polydimethylsiloxane (PDMS) substrate with a microcavity to generate optical interference. The cavity structure was fabricated by transferring the submicron-thick bilayer to the highly adhesive PDMS substrate. The detection of human serum albumin antigen at a concentration of $1 \mathrm{pg} / \mathrm{mL}$ was achieved by antigen-antibody reaction using simultaneous optical and electrical measurements. In addition, the minimum detectable displacement of the optical interferometric surface stress sensor was determined to be $42.6 \mathrm{pm}$, representing an 11.7-fold improvement compared with conventional cantilever-based surface stress sensors.

\section{Introduction}

MEMS-based sensors can detect physical quantities such as pressure, acceleration, and surface stress in real time, contributing to the Internet of Things (IoT). As a sensor for measuring physical quantities as well as biomolecules and chemical substances, MEMS-based sensors that operate on the basis of the mechanical response due to molecular adsorption can be used to detect various substances. This type of sensor can measure mechanical and electrical changes induced by adsorption in real time. In particular, a method for accurately and quickly inspecting biomolecules including diseased proteins (biomarkers), gas molecules in blood tests, and exhaled gases has attracted attention as a promising simple diagnostic method for various diseases.

\footnotetext{
*Corresponding author: e-mail: takahashi@ee.tut.ac.jp

${ }^{\dagger}$ These authors contributed equally to this work.

https://doi.org/10.18494/SAM.2019.2247
} 
Technologies for detecting gas molecules and biomolecules on miniaturized sensor elements and arrayed using semiconductor microfabrication technology have been developed for targets such as DNA, ${ }^{(1,2)}$ tumor markers, ${ }^{(3,4)}$ Escherichia coli,${ }^{(5)}$ viruses, ${ }^{(6,7)}$ and gas molecules. ${ }^{(8-13)}$ It is expected that the parallel detection of different molecules would allow for prompt verification with such sensors. ${ }^{(2,8,9)}$ A MEMS-based sensor featuring a system for detecting a static deflection of the dynamic system and a micromechanical structure to detect changes in mechanical resonance frequency has been proposed. ${ }^{(14-19)}$ A functional film that specifically absorbs a molecule is formed on a silicon resonator and the change in resonance frequency after the absorption of the molecule is measured. Piezoresistive sensors employ a piezoresistor embedded in a movable silicon cantilever, which are subjected to resonant vibration, and frequency detection is achieved by the vibration of the piezoresistive cantilever. ${ }^{(20,21)}$ However, in this vibration-type chemical sensor, an oscillation circuit is required for each sensor for frequency analysis, complicating the system. On the other hand, surface stress sensors that detect static deformation due to molecular adsorption use the operating principle of surface stress change detection arising from the repulsive force of the adsorbed molecules. A surface stress sensor typically exhibits less performance deterioration due to viscosity than a resonance sensor. A surface stress sensor must be electrically detected in a chip to detect changes in electrostatic capacitance, so a piezoresistive element is typically integrated in the movable film to detect changes in membrane resistance. However, conventional surface stress sensors respond to the displacement of the membrane in inverse proportion to the electrostatic type and proportionally to the piezoresistance type, preventing the improvement of signal conversion efficiency. We previously reported a novel fabrication method for optical interference-type surface stress sensors ${ }^{(22)}$ to improve their signal conversion efficiency. In addition, MEMS optical interference sensors contain unique features in their structure and the sensor section is a photodiode. Therefore, the sensor can be integrated with a CMOS image sensor. The voltage response of the sensor amplified by the buffer can be outputted using CMOS image sensor technology. ${ }^{(23)}$ Therefore, it is possible to use imaging to measure gas distributions and for the multidetection of biomolecules. Compared with conventional MEMS-type sensors, the developed system showed that the signal conversion efficiency can be improved using a steep slope of optical interference characteristics and analytically improved by two orders of magnitude. ${ }^{(22)}$

Moreover, the deflection detection limit is an important parameter of surface stress sensors. By detecting tiny deflections, molecular adsorption at low concentrations can be measured. It has been reported that the displacement detection limit of an optical interferometry-based strain sensor is an order of magnitude superior to that of conventional cantilever sensors. ${ }^{(9,24-27)}$ In this study, we improved the displacement detection limit of a surface stress sensor using current conversion by optical interference. Using this detection technique, a small surface stress caused by molecular adsorption can be measured, leading to the detection of low concentrations of target molecules. We also compared the detection limit of the system developed herein with that of a cantilever sensor. 


\section{Design and Fabrication}

\subsection{Device structure}

The surface stress sensor consists of a suspended parylene-C membrane attached to a polydimethylsiloxane (PDMS) substrate with a cavity-sealed structure formed by dry transfer. Optical interference is provided by the cavity formed under the suspended membrane, which is used for signal transducing from mechanical deflection to transmission/reflection intensity using a single wavelength exposed from the outside. For the immobilization of a receptor to selectively bind target molecules, polymethyl methacrylate (PMMA) is coated on the suspended parylene-C membrane. Since PDMS is highly adhesive to the PMMA/parylene-C nanosheet as a movable film leading to the permanent bonding of the transferred PMMA/parylene-C sheet at room temperature, PDMS was used as the substrate.

\subsection{Working principle}

The operation principle of the optical interferometric surface stress sensor is shown schematically in Fig. 1. The membrane deforms upon the introduction of surface stress associated with the antigen-antibody reaction. This response is caused by the immobilization of the antibody by treating the surface of the movable membrane and the subsequent binding of an antigen to the immobilized antibody. When the antigen-antibody reaction occurs, the membrane deforms and a spectral change occurs. When this change is detected by the

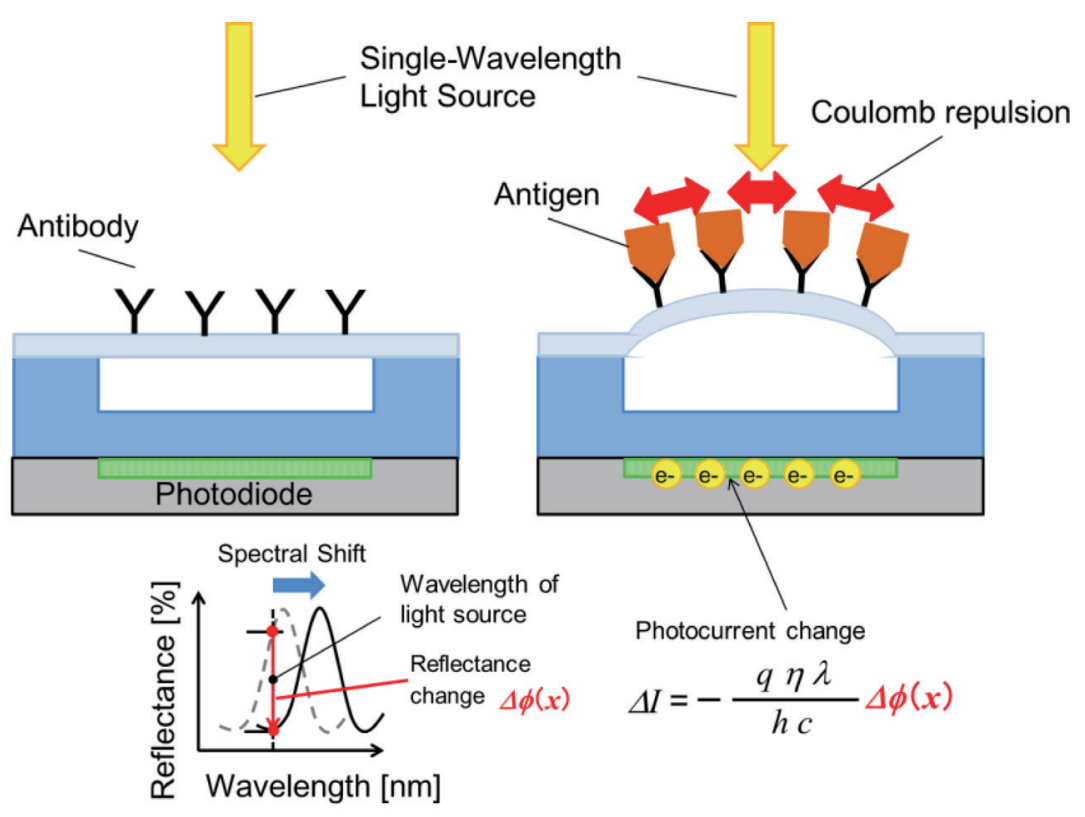

Fig. 1. (Color online) Deflection model of the movable film due to molecular binding. 
photodiode, an electrical output is generated. The displacement detection lower limit of the current measurement detected by the photodiode was investigated herein. When the noise content is the dark current of the photodiode, the signal-to-noise (SN) ratio can be described as

$$
S N=I_{p} / I_{\text {dark }}
$$

where $I_{p}$ is the photocurrent measured using a photodiode per unit time and $I_{\text {dark }}$ is the dark current of the photodiode. The displacement detection lower limit $D_{\text {lower }}$ can be expressed using the following equation:

$$
D_{\text {lower }}=D_{p} / S N
$$

where $D_{p}$ is the deformation amount per unit time.

\subsection{Design}

For the estimation of the sensitivity of the proposed sensor, we calculate the deflection for surface stress. The static deflection $\Delta z$ is determined by the following equation:

$$
\Delta z \propto \frac{D^{2}(1-v)}{E t^{2}} \Delta \sigma
$$

where $D$ is the diameter, $v$ is the Poisson ratio, $E$ is Young's modulus, $t$ is the film thickness, and $\sigma$ is the surface stress. From this equation, the sensitivity of the surface stress sensor is inversely proportional to Young's modulus and the square of the film thickness. Therefore, parylene-C with Young's modulus two orders of magnitude lower than that of silicon is used. In addition, the film thickness of parylene- $\mathrm{C}$ can be controlled on the submicron order. From these features, the sensitivity of the proposed sensor can be expected to be two orders of magnitude greater than that of a conventional cantilever-based sensor. ${ }^{(28)}$

\subsection{Fabrication}

Figure 2 shows the fabrication of the optical interferometric surface stress sensor with a PDMS substrate. First, parylene-C was vapor-deposited on the silicon substrate that was coated with a surfactant. Subsequently, PMMA was spin-coated on the parylene-C layer, followed by soaking in ultrapure water to peel off the sheet. The thickness of this parylene-C sheet was $100 \mathrm{~nm}$.

To prepare the PDMS substrate, SU-8 was spin-coated onto the silicon substrate, followed by patterning by photolithography to produce a mold. The PDMS substrate with microcavities was then prepared by peeling the PDMS coated on the SU- 8 mold. The cavity diameter and depth were designed to be 50-300 and $3.5 \mu \mathrm{m}$, respectively. The nanosheet was then dry-transferred 


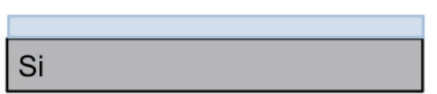

(a) Deposition Parylene-C

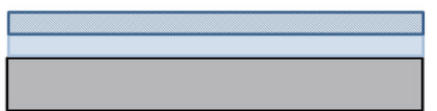

(b) Spin coating of PMMA

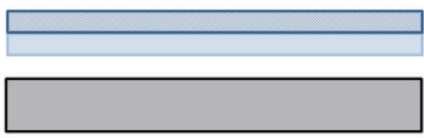

(c) Release sheet in DI water

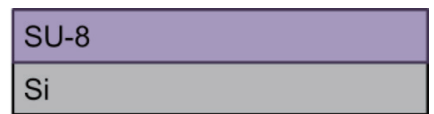

(d) Spin coating of SU-8

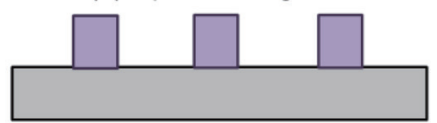

(e) Lithography

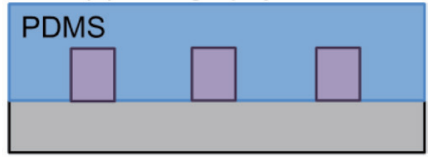

(f) Coating PDMS

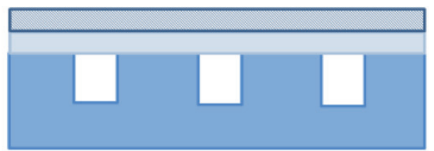

(g) Peeling PDMS, dry transfer of Parylene

nanosheet, and UV-ozone treatment

Fig. 2. (Color online) Fabrication of a Fabry-Perot interferometric surface stress sensor.

on the PDMS substrate with microcavities. Finally, the PMMA surface was oxidized using a UV ozone cleaner for the immobilization of biomolecules, as the UV radiation generates carboxylic groups on the PMMA surface. ${ }^{(29)}$ The substrate material can be replaced by a silicon substrate with a prepatterned cavity instead of PDMS.

\section{Experimental Results}

Before performing biometric measurements, the surface of the prepared surface stress sensor was subjected to solution treatment for antibody immobilization on the suspended membrane for selective molecular detection. To bind the amino group of the antibody to the carboxyl group of the oxidized PMMA layer, treatment with 1-ethylo-3-(3-dimethylaminopropyl)carbodiimide (EDC)/N-hydroxysuccinimide (NHS) solution was performed for $30 \mathrm{~min}$ and subsequently rinsed with PBS. Next, the sensor was dipped in a solution of human serum albumin (HSA) antibody for $2 \mathrm{~h}$, followed by washing with PBS, and subsequently immersed in a solution of bovine serum albumin (BSA) as a blocking agent for $2 \mathrm{~h}$. The antibody-immobilized sensor chip was then immersed in $1.9 \mathrm{~mL}$ of PBS for $30 \mathrm{~min}$, and it was confirmed that the movable film of the surface stress sensor was stabilized. In addition, to investigate the physical effect of dropping the solution, the deflection of the membrane was measured upon the dropwise addition of $0.1 \mathrm{~mL}$ of PBS, and it showed no response. After the above pretreatment, an HSA antigen solution was added dropwise to a final concentration of $1 \mathrm{pg} / \mathrm{mL}-10 \mathrm{ng} / \mathrm{mL}$ to evaluate the antigen-antibody reaction. 
Figure 3(a) shows typical reflection spectra from the optical interferometric surface stress biosensor with a diameter of $50 \mu \mathrm{m}$ immersed in an HSA solution with a final concentration of $10 \mathrm{ng} / \mathrm{mL}$. Peak positions were observed to redshift after dropping the HSA solution, which means that the PMMA/parylene-C membrane deforms in the upper direction in a liquid. We assumed that the membrane was deformed by the surface stress induced through the molecular binding due to the antigen-antibody reaction. We also evaluated the concentration dependence of the biosensor on the antigen-antibody reaction. The time course of the specific peak position after dropping the HSA antigen solution is shown in Fig. 3(b). It can be confirmed that the membrane deflection changes depending on the concentration. As a response to nonspecific adsorption, the membrane deflection in a streptavidin solution with a concentration of $1 \mathrm{ng} / \mathrm{mL}$ was evaluated. The membrane deflection derived from the antigen-antibody reaction to $1 \mathrm{pg} / \mathrm{mL}$ HSA was larger than that of physical adsorption. In addition, this response to physical adsorption can be distinguished by comparison with a control sensor without an antibody. Moreover, the differential operation with the implementation of the control sensor and comparator circuit can remove not only the physical adsorption of molecules but output fluctuations caused by pressure and incident light fluctuations. ${ }^{(30)}$

In the experimental setup shown in Fig. 4, the surface stress sensor simultaneously measured the reflection spectrum of the optical interferometer treated with the HSA antigen and the photocurrent measured with a photodiode chip (SM05PD2A, Thorlabs). For the current measurement, a $680 \mathrm{~nm}$ laser exposed to the sensor was prepared. Here, when the laser light enters the spectrometer, the output signal at the laser wavelength saturates and spectrometry is not possible. Since the spectroscopic measurements were performed by attaching a shortpass filter to the spectrometer (USB4000 Ocean Optics) to remove the probe laser at $680 \mathrm{~nm}$ for current measurement, the reflection spectrum was measured from 400 to $650 \mathrm{~nm}$. By observing the spectrum shift in the visible range, it was possible to demonstrate the validity of the photocurrent change by irradiation at $680 \mathrm{~nm}$. To prevent the evaporation of the solution, the experiment was performed in a petri dish.

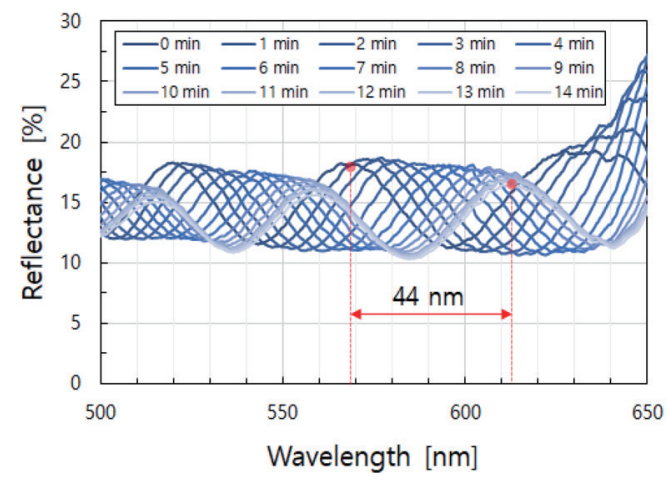

(a)

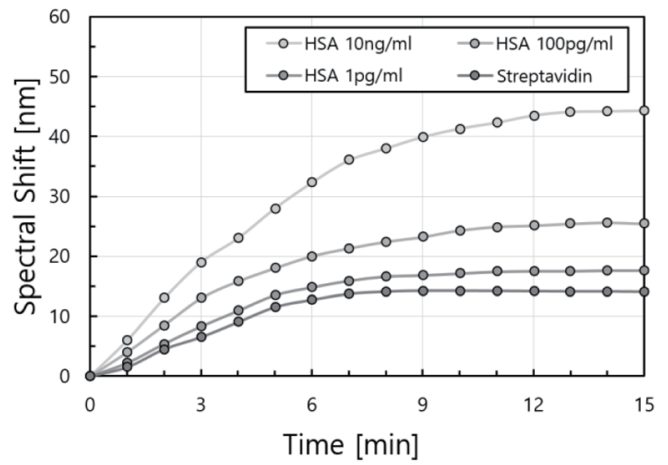

(b)

Fig. 3. (Color online) (a) Typical reflection spectra from the optical interferometric surface stress biosensor with a diameter of $50 \mu \mathrm{m}$ immersed in an HSA solution with a final concentration of $10 \mathrm{ng} / \mathrm{mL}$. (b) Time course of the specific peak position corresponding to the concentration of HSA. 


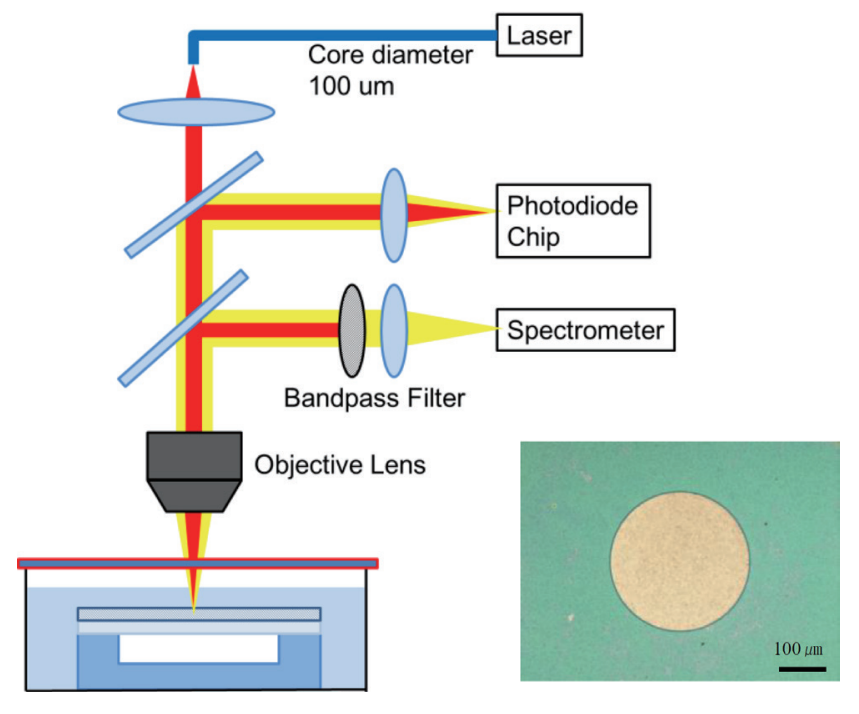

Fig. 4. (Color online) Schematic diagram of the experimental setup.

For the evaluation of the minimum detection limit of membrane deflection, the reflection spectrum in a $1 \mathrm{pg} / \mathrm{mL}$ HSA solution was measured using an optical interferometric sensor with a silicon substrate because the reflectance of the surface of silicon as the bottom halfmirror of the interferometer is well-known. Figure 5(a) shows reflection spectra of the optical interferometric sensor with a diameter of $300 \mu \mathrm{m}$ corresponding to the treatment time in the HSA solution. The peak positions were found to redshift and the movement became gradually slower. Figure 5(b) shows the time course of the movement of the spectrum in which a peak was observed around $550 \mathrm{~nm}$ immediately after the start of measurement. It was observed that the rate of shift was the highest and gradually saturated. To estimate the membrane deflection, curve fitting was carried out for different air gaps of the Fabry-Perot interferometer, as shown in Fig. 5(c). The analysis parameters were set to 100 -nm-thick parylene-C, 150-nm-thick PMMA, and a variable air gap between 5.48 and $5.582 \mu \mathrm{m}$. The fitting curves were obtained using optical analysis software (RSOFT DiffractMOD). Because the interference peaks were determined by the air gap, optical simulation was performed to fit the peak positions. Although reflectance by the optical simulation did not coincide with the experimental values, it depended on the reflectance of the mirror surfaces of the Fabry-Perot interferometer. Thus, it can be considered that the reflectance of the PMMA and solution interface decreased owing to molecular adsorption. On the other hand, peak positions were in good agreement with the experimental values. Therefore, it was suggested that the membrane was deformed by $102 \mathrm{~nm}$ in $300 \mathrm{~s}$ by the antigen-antibody reaction.

Figure 6 shows the output photocurrent associated with the spectral shift in the reflection spectrum. The photocurrent was measured with a picoammeter (6485 Picoammeter, Keithley) and the photocurrent change was obtained according to the reflectance at $680 \mathrm{~nm}$ accompanying the spectrum shift. The photocurrent gradually increased and saturated, which was consistent with the reflection spectrum change mentioned above. 


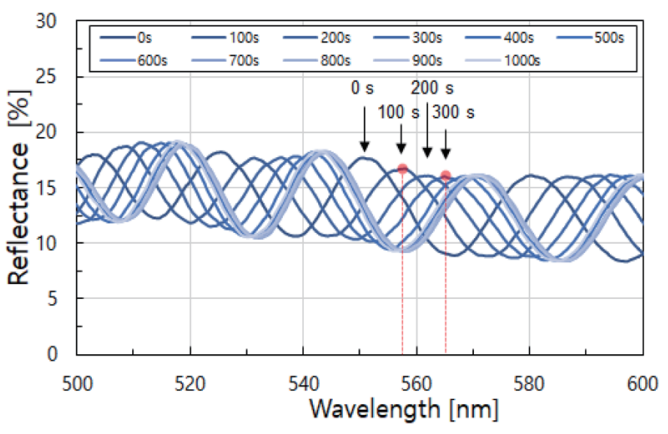

(a)

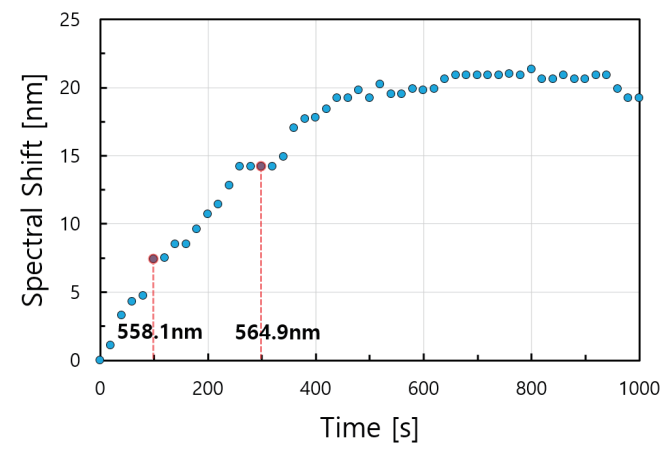

(b)

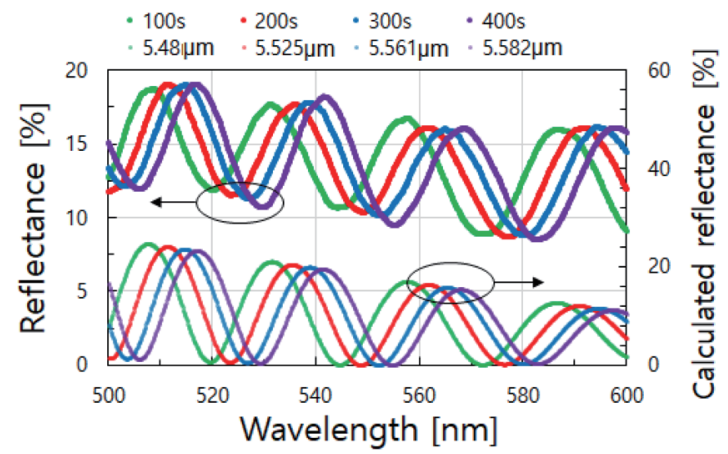

(c)

Fig. 5. (Color online) (a) Change in the reflection spectrum of Fabry-Perot interferometric sensor in $1 \mathrm{pg} / \mathrm{mL}$ HSA. (b) Time course of the movement of the spectrum in which a peak was observed around $550 \mathrm{~nm}$ immediately after the start of measurement. (c) Fitting curves used to estimate membrane deflection by rigorous coupled-wave analysis.

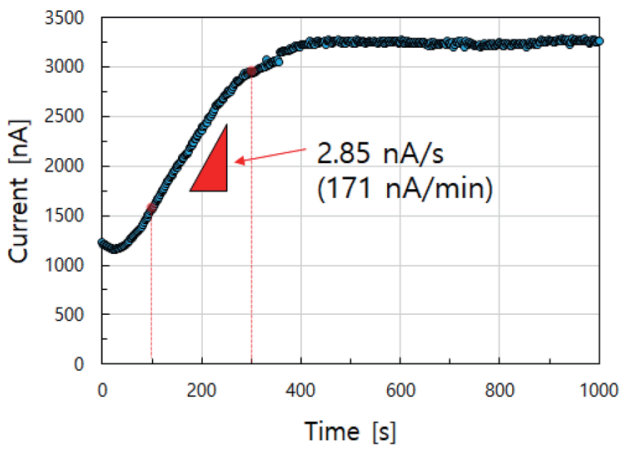

Fig. 6. (Color online) Time course result of photocurrent from a photodiode with exposure to $680 \mathrm{~nm}$ light source.

\section{Discussion}

The minimum detection limit of membrane deflection was determined from the results presented herein. In the proposed optical interferometry for deflection measurement, the membrane deflection is transduced to the reflected light intensity change associated with the interference peak shift, which is not a discrete change but a continuous change. Therefore, 
LOD for displacement can be obtained by the extrapolation of the measured current. First, focusing on the two points at 100 and $300 \mathrm{~s}$ in Fig. 6, the current change of the photodiode per unit time was determined to be $171 \mathrm{nA}$. Since the noise was $0.3 \mathrm{nA}$, as determined by the dark current of the photodiode, the SN ratio was 570. The amount of deformation per unit time was also calculated from Fig. 5(c) to be $24.3 \mathrm{~nm}$. When this value was divided by the SN ratio, the minimum detection limit for the developed sensor was determined to be $42.6 \mathrm{pm}$, which is 11.7 times greater than that of a typical cantilever-type sensor. ${ }^{(9)}$ In addition, the nanomechanical deflection associated with surface stress was analyzed by a finite element method using COMSOL and compared with that of the silicon cantilever sensor shown in Fig. 7. The minimum detection limit of the surface stress of the cantilever sensor with dimensions of 500 $\mu \mathrm{m}$ length, $100 \mu \mathrm{m}$ width, and $1 \mu \mathrm{m}$ thickness was $0.15 \mathrm{mN} / \mathrm{m}$, with a displacement detection limit of $500 \mathrm{pm} .{ }^{(9)}$ On the other hand, the detection limit of the surface stress of the optical interferometric surface stress sensor was $0.4 \mu \mathrm{N} / \mathrm{m}$, as determined by COMSOL calculation, and the experimentally obtained displacement limit was improved by a factor of 11.7.

In terms of target concentration response, the detection limit of a typical conventional cantilever sensor is summarized in Table 1. Although the conventional cantilever sensor detection limit is $0.2 \mathrm{ng} / \mathrm{mL}$, the proposed sensor showed sufficient response for a concentration of $1 \mathrm{pg} / \mathrm{mL}$, representing a sensitivity 200 times greater than that of the cantilever sensor owing to its excellent stress sensitivity by reducing the stiffness of the suspended membrane by using the thin parylene- $\mathrm{C}$ film and high displacement resolution using optical interferometry. In addition, the detection capability of the surface stress biosensor depends on the minimum detection limit of the deflection as well as the biointerface including the density of a cross linker and the affinity of a receptor. Therefore, the minimum detection limit of the molecular concentration can be improved by optimizing the immobilization density of the antibody.

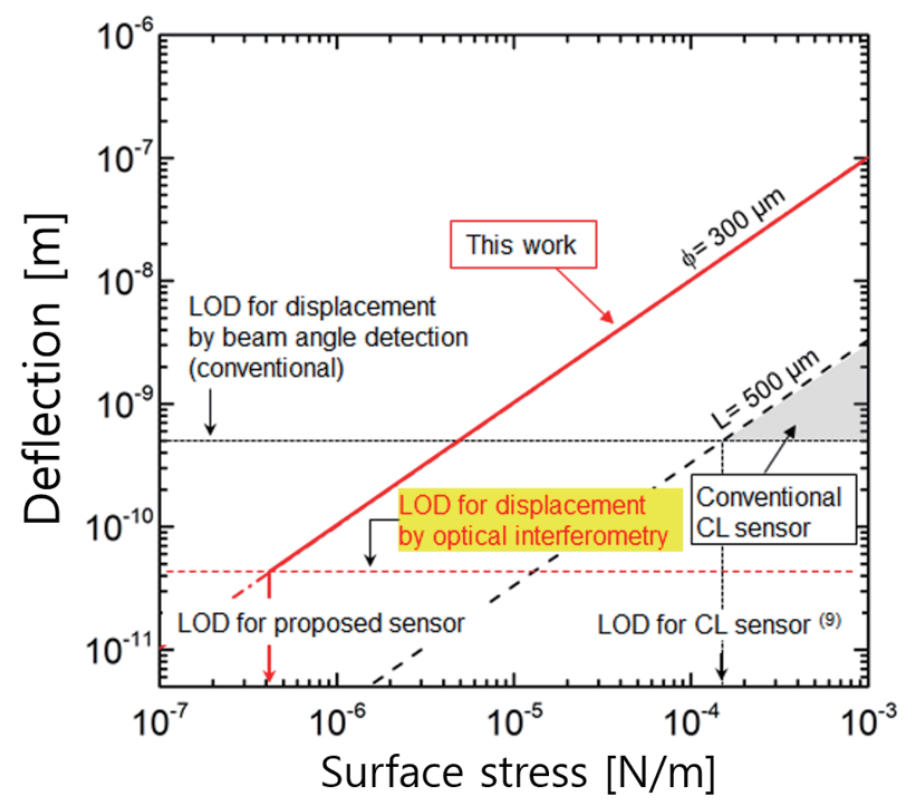

Fig. 7. (Color online) Comparison of optical interferometric sensor and silicon cantilever sensors by surface stress analysis. 
Table 1

Comparison of limits of detection.

\begin{tabular}{lccc}
\hline Analyte & Limit of detection $(\mathrm{ng} / \mathrm{mL})$ & Device type & Ref. \\
\hline PSA & 10 & Cantilever & $(24)$ \\
ALCAM & 10 & Cantilever & $(25)$ \\
PSA & 10 & Cantilever & $(26)$ \\
PSA & 0.2 & Cantilever & $(27)$ \\
\hline
\end{tabular}

\section{Conclusions}

Herein, we reported an improved displacement detection limit using a novel label-free surface stress biosensor by optical interferometry. The proposed sensor consists of a cavity structure formed by an oxidized PMMA/parylene-C membrane and PDMS substrate, and the sealed cavity was fabricated by transferring a sub-micron-thick PMMA/parylene-C membrane to a highly adhesive PDMS. The spectrum was measured by membrane deflection and the electrical output from the diode chip was simultaneously obtained. This optical interferometric surface stress sensor showed a displacement detection limit 11.7 greater than that of a previously reported silicon cantilever sensor, ${ }^{(9)}$ and its limit of protein detection was 200 times greater than those of other cantilever type sensors. ${ }^{(24-27)}$

\section{Acknowledgments}

This work was supported in part by the Precursory Research for Embryonic Science and Technology (PRESTO) (15655927) from the Japan Science and Technology Agency, a Grant-inAid for Young Scientists (18K14106) from the Japan Society for the Promotion of Science, and Toukai Foundation for Technology.

\section{References}

1 J. R. Barnes, R. J. Stephenson, M. E. Welland, C. Gerber, and J. K. Gimzewski: Nature 372 (1994) 79.

2 R. McKendry, J. Y. Zhang, Y. Arnt, T. Strunz, M. Hegner, H. P. Lang, M. K. Baller, U. Certa, E. Meyer, H. J. Guntherodt, and C. Gerber: Proc. Natl. Acad. Sci. U.S.A. 99 (2002) 9783.

3 J. K. Gimzewski, C. Gerber, E. Meyer, and R. R. Schlittler: Chem. Phys. Lett. 217 (1994) 589.

4 H. P. Lang, M. K. Baller, R. Berger, C. Gerber, J. K. Gimzewski, F. M. Battiston, P. Fornaro, J. P. Ramseyer, E. Meyer, and H. J. Guntherodt: Anal. Chim. Acta 393 (1999) 59.

5 R. Berger, E. Delamarche, H. P. Lang, C. Gerber, J. K. Gimzewski, E. Meyer, and H. J. Guntherodt: Science 276 (1997) 2021.

6 J. Fritz, M. K. Baller, H. P. Lang, H. Rothuizen, P. Vettiger, E. Meyer, H. J. Guntherodt, C. Gerber, and J. K. Gimzewski: Science 288 (2000) 316.

7 G. H. Wu, R. H. Datar, K. M. Hansen, T. Thundat, R. J. Cote, and A. Majumdar: Nat. Biotechnol. 19 (2001) 856.

8 G. Yoshikawa, H. P. Lang, T. Akiyama, L. Aeschimann, U. Staufer, P. Vettiger, M. Aono, T. Sakurai, and C. Gerber: Nanotechnology 20 (2009) 015501.

9 G. Yoshikawa, T. Akiyama, S. Gautsch, P. Vettiger, and H. Rohrer: Nano Lett. 11 (2011) 1044.

10 K. K. Park, H. Lee, M. Kupnik, O. Oralkan, J. P. Ramseyer, H. P. Lang, M. Hegner, C. Gerber, and B. T. Khori-Yakub: Sens. Actuators, B 160 (2011) 1120.

11 H. J. Lee, K. K. Park, M. Kupnik, and B. T. Khuri-Yakub: Sens. Actuators, B 174 (2012) 87.

12 D. M. Karabacak, S. H. Brongersma, and M. C. Calama: Lab Chip 10 (2010) 1976. 
13 J. Pettine, M. Patrascu, D. M. Karabacak, M. Vandecasteele, V. Petrescu, S. H. Brongersma, M. C. Calama, and C. Van Hoof: Sens. Actuators, A 189 (2013) 496.

14 N. Backmann, C. Zahnd, F. Huber, A. Bietsch, A. Pluckthun, H. P. Lang, H. J. Guntherodt, M. Hegner, and C. Gerber: Proc. Natl. Acad. Sci. U.S.A. 102 (2005) 14587.

15 J. Zhang, H. P. Lang, F. Huber, A. Bietsch, W. Grange, U. Certa, R. McKendry, H.-J. Guntherodt, M. Hegner, and C. Gerbe: Nat. Nanotechnol. 1 (2006) 214.

16 D. A. Raorane, M. D. Lim, F. F. Chen, C. S. Craik, and A. Majumdar: Nano Lett. 8 (2008) 2968.

17 M. K. Ghatkesar, H. P. Lang, C. Gerber, M. Hegner, and T. Braun: PLoS One 3 (2008) e3610-1.

18 R. Mukhopadhyay, V. V. Sumbayev, M. Lorentzen, J. Kjems, P. A. Andreasen, and F. Besenbacher: Nano Lett. 5 (2005) 2385.

19 J. L. Arlett, J. R. Maloney, B. Gudlewski, M. Muluneh, and M. L. Rouke: Nano Lett. 6 (2006) 1000.

20 M. Tortonese, R. C. Barrett, and C. F. Quate: Appl. Phys. Lett. 62 (1993) 834.

21 L. Aeschimann, A. Meister, T. Akiyama, B. W. Chui, P. Niedermann, H. Heinzelmann, N. F. De Rooij, U. Staufer, and P. Vettiger: Microelectron. Eng. 83 (2006) 1698.

22 K. Takahashi, R. Ozawa, H. Oyama, M. Futagawa, F. Dasai, M. Ishida, and K. Sawada: Proc. IEEE Int. Electron Devices Meeting (IEDM, 2012) 553-556.

23 S. Maruyama, T. Hizawa, K. Takahashi, and K. Sawada: MDPI Sensors 18 (2018) 138. https://doi.org/10.3390/ s18010138

24 J. Bausells: Microelectron. Eng. 145 (2015) 9.

25 M. G. von Muhlen, N. D. Brault, S. M. Knudsen, S. Jiang, and S. R. Manalis: Anal. Chem. 82 (2010) 1905. https://doi.org/10.1021/ac9027356

26 K. W. Wee, G. Y. Kang, J. Park, J. Y. Kang, D. S. Yoon, J. H. Park, and T. S. Kim: Biosens. Bioelectron. 20 (2005) 1932.

27 G. Wu, R. H. Datar, K. M. Hansen, T. Tundat, R. J. Cote, and A. Majumdar: Nat. Biotechnol. 19 (2001) 856.

28 K. Takahashi, H. Oyama, N. Misawa, K. Okumura, M. Ishida, and K. Sawada: Sens. Actuators, B 188 (2013) 393. https://doi.org/10.1016/j.snb.2013.06.106

29 M. Rowinska, S. M. Kelleher, F. Soberon, A. J. Ricco, and S. Daniels: J. Mater. Chem. B 3 (2015) 135. https:// doi.org/10.1039/c4tb01748j

30 S. Maruyama, T. Hizawa, K. Takahashi, and K. Sawada: 43rd Int. Conf. Micro and Nanoengineering (MNE, 2017) PO084.

\section{About the Authors}

Satoshi Maruyama received his Ph.D. degree in electrical engineering from the University of Tokyo, Tokyo, Japan, in 2013. From 2013 to 2014, he was a postdoctoral researcher at Research Center for Advanced Science and Technology (RCAST), The University of Tokyo. From 2014 to 2016, he was a postdoctoral researcher at VLSI Design and Education Center (VDEC), The University of Tokyo. Since April 2016, he has been a project assistant professor at Toyohashi University of Technology. His research interests are in integrated MEMS for chemical and biological sensing processes. (maruyama@ee.tut.ac.jp)

Yong-Joon Choi received his B.S. and M.S. degrees in information technology and electronic education and bio-electronic engineering from the Andong National University, Gyeongbuk, Korea in 2010 and 2013, respectively, and his Ph.D. degree in electrical and electronic information engineering, Toyohashi University of Technology, Aichi, Japan, in 2017. Since 2017, he has been a postdoctoral researcher at the Department of the Electrical and Electronic Information Engineering of Toyohashi University of Technology. His research interests focus on the development of optical biosensors and bio-MEMS. (choi@int.ee.tut.ac.jp) 
Kazuhiro Takahashi received his B.S. degree in mechanical engineering from Nagoya University, Japan in 2003 and his M.S. and Ph.D. degrees in electrical engineering from The University of Tokyo, Japan, in 2005 and 2008, respectively. From 2008 to 2009, he was a postdoctoral researcher with the Institute of Industrial Science (IIS), The University of Tokyo. From 2009 to 2013, he was an assistant professor of electrical and electronic information engineering at Toyohashi University of Technology. Since 2013, he has been a tenure track lecturer of electrical and electronic information engineering at Toyohashi University of Technology. His research interest is in the development of CMOS-MEMS sensors and actuators for optical and biological applications. (takahashi@ee.tut.ac.jp)

Kazuaki Sawada was born in Kumamoto, Japan, in 1963. He received his B.A. and M.S. degrees in electrical and electronic engineering and his Ph.D. degree in system and information engineering from Toyohashi University of Technology, Aichi, Japan, in 1986, 1988, and 1991, respectively. From 1991 to 1998, he worked as a research associate in the Research Institute of Electronics, Shizuoka University, Shizuoka, Japan. In 1998, he joined the Department of Electrical and Electronic Engineering, Toyohashi University of Technology, Aichi, Japan, and he is currently a professor and director of Electronics-Inspired Interdisciplinary Research Institute (EIIRIS). In 2005, he was a guest researcher with the Technical University of Munich, Munich, Germany. His current research interests focus on the development of ultrahigh-sensitivity biosensing and microfluidic devices. (sawada@ee.tut.ac.jp) 